\title{
Evaluación de recubrimientos de quitosano sobre cuerpos porosos de hidroxiapatita
}

\author{
Evaluation of chitosan coatings on hydroxyapatite scaffolds.
}

\author{
N. Mesa-Ospina ; D. M. Escobar-Sierra iD
}

\begin{abstract}
Resumen- Los defectos óseos han sido tratados implantando tejidos autólogos o alogénicos, sin embargo, el éxito de estos implantes depende de la biocompatibilidad, fijación con el tejido, características físicoquímicas y las complicaciones vinculadas al uso de injertos, como la limitada cantidad de hueso a extraer, la morbilidad en el lugar de extracción y los potenciales riesgos por infección; por ello, surge la Ingeniería de Tejidos, donde las propiedades superficiales y mecánicas de los biomateriales empleados juegan un papel importante en la interacción materialtejido. En esta investigación se desarrollaron cuerpos porosos de hidroxiapatita por gel-casting y se recubrieron con quitosano a diferentes concentraciones, con el fin de verificar la influencia del recubrimiento en las propiedades mecánicas y de estabilidad de los cuerpos fabricados, comparándolas con las del hueso para intentar mejorar los requerimientos de los implantes. Para ello, se evaluó la morfología del poro, la resistencia a la compresión y la degradabilidad de los cuerpos con y sin recubrimiento. Los resultados mostraron que se logra mejorar las propiedades y la estabilidad en condiciones fisiológicas, lo que los hace aptos para usar en regeneración de tejido óseo.
\end{abstract}

Palabras clave - Cuerpos porosos, Gel-casting, Hidroxiapatita, Quitosano, Recubrimientos, Resistencia mecánica, Tratamientos médicos.

Abstract - Bone defects have been treated implanting autologous, allogeneic or tissues, however, the success of these procedures depends of biocompatibility, fixation with the tissue, physicochemical characteristics and the complications related to the extraction of autologous grafts, such as the limited amount of bone to be extracted, the morbidity in the place of extraction and the potential risks of infectious nature, therefore, arise the Tissue Engineering where the superficial and mechanical properties of the biomaterials used play an important role in material-tissue interaction. In this investigation, porous bodies of hydroxyapatite were developed by gel-casting and they were coated with chitosan at different concentrations to verify the influence of coating on the mechanical properties and stability of the manufactured porous bodies, comparing the properties obtained with of the bone for trying to improve the requirements for implants.

For those, pore morphology, compressive strength and degradability the porous bodies were evaluated with and without coating, and the results showed that the porous bodies have properties of mechanical strength and adequate morphology for use in regeneration of bone tissue, confirming considerably improvements in these and demonstrating an effective and efficient shielding of the porous bodies of HA under physiological conditions. higher concentration of chitosan with respect to the porous bodies of uncoated HA.

Index Terms - Chitosan, Coating, Gel-casting, Hydroxyapatite, Mechanical strength, Scaffolds, Medical treatment.

\section{INTRODUCCIÓN}

$\left\lceil\int^{N}\right.$ $\mathrm{N}$ reemplazo óseo debe imitar al tejido respondiendo a sus requerimientos de forma $y$ funcionamiento, promoviendo una respuesta adecuada del sistema biológico sin inducir ningún tipo de infección, para una mejor interacción con el tejido. Generalmente, se utiliza tejido óseo extraído del propio paciente, por ser el que facilita mejor su recuperación. Sin embargo, la cantidad de tejido que puede ser auto trasplantado es limitada y en ocasiones, es necesario realizar otra intervención quirúrgica, ya que puede presentarse infección o rechazo [1].

Debido a esto, surge la Ingeniería de Tejidos (IT) como una disciplina que integra factores bioactivos y plataformas de soporte (cuerpos porosos) que pueden obtenerse a partir de biomateriales y que proporcionan no sólo apoyo mecánico e integridad al hueso, sino que permiten el crecimiento adecuado del tejido natural [2].

Este manuscrito fue enviado el 3 de diciembre del 2018 y aceptado 15 de marzo, de 2019. Este Proyecto fue financiado por la Universidad de Antioquia, Medellín.

N. Mesa-Ospina. Es Bioingeniera, Magister en Ingeniería de Materiales de la Universidad de Antioquia, Medellín, Actualmente trabaja en Interventional Medical Products. (e-mail: mesanatali@gmail.com).
D. M. Escobar-Sierra es Ingeniera Metalúrgica, Ph.D. en Ciencias Químicas. Es Profesora Titular de la Facultad de Ingeniería de la Universidad de Antioquia. Medellín, Colombia. (e-mail: marcela.escobar@udea.edu.co). 
Para tal fin se han planteado diferentes técnicas de fabricación de plataformas utilizando diversos materiales, entre los que sobresale la Hidroxiapatita (HA), por su gran capacidad de enlazarse al tejido óseo [3], aunque su baja resistencia mecánica la limita en ciertas aplicaciones [4]; por lo que se hace necesario evaluar técnicas adicionales para mejorar su resistencia mecánica. Dado que la matriz ósea está compuesta por una fase dura inorgánica (minerales tipo fosfato de calcio) y una orgánica (proteínas y colágeno), cuya asociación otorga la resistencia necesaria para soportar las cargas mecánicas, se ha propuesto simular dicha estructura desarrollando cuerpos tridimensionales porosos compuestos de cerámicos y polímeros, buscando asociar las excelentes propiedades osteoconductivas de los cerámicos, a su vez la flexibilidad y degradabilidad de los polímeros [5, 6].

Conociendo la biocompatibilidad, bioadhesividad y capacidad de bioestimulación del quitosano (biopolímero natural extraído de caparazón de crustáceos), se ha planteado en esta investigación, evaluar la influencia de este biopolímero como recubrimiento superficial, en el incremento de las propiedades mecánicas de los cuerpos porosos de hidroxiapatita, para evaluar su potencial aplicación en Ingeniería de Tejido Óseo.

\section{METODOLOGÍA}

\section{A. Materiales.}

Los scaffolds fueron producidos mediante la técnica de gelcasting usando reactivos de grado analítico: Hidroxiapatita (HA) comercial (Strem Chemicals) como precursor de sólidos, monómeros de Acrilamida (Merck) para la reacción de polimerización In situ, Persulfato de amonio (Aldrich) como agente iniciador, y Tetrametiletilendiamina-TEMED (Merck) como catalizador; Ácido metacrílico (Aldrich) como dispersante, Tergitol ( $\alpha$ químicos Ltda) y Dodecil sulfato de sodio (J.T Baker), como surfactantes y Bisacrilamida (Merk) como entrecruzante.

Para las soluciones de recubrimiento se utilizó quitosano comercial (Sigma-Aldrich, \%DD >75\%) y ácido acético glacial (Panreac) como disolvente.

\section{B. Fabricación de los cuerpos porosos de HA.}

La preparación de los cuerpos porosos se realizó mediante la técnica de gel-casting descrito por González [3] utilizando un porcentaje de sólidos del $50 \%$ de HA. El procedimiento se realizó en una cámara de vacío con atmósfera de nitrógeno para evitar reacción con el oxígeno, lo cual inhibe la polimerización de los monómeros.

Inicialmente se mezcló la acrilamida con agua destilada durante 3 minutos bajo agitación magnética, y posteriormente se adicionó el polvo de HA y el persulfato de amonio, y se continuó la homogenización por 10 minutos hasta romper los aglomerados de las partículas del polvo de HA.

Pasado este tiempo, se adicionó la bisacrilamida y el ácido metacrílico bajo agitación mecánica y seguidamente se adicionó el TEMED, el tergitol y dodecil sulfato de sodio bajo agitación vigorosa hasta lograr espuma, esta solución finalmente se distribuyó en los moldes dejándolos dentro de la cámara de vacío durante 30 minutos para completar el proceso de polimerización in situ.

Una vez alcanzada completamente la polimerización, los moldes fueron secados a temperatura ambiente por 24 horas y luego se pasaron a una estufa a $70^{\circ} \mathrm{C}$ por 15 horas para eliminar la humedad del interior y proporcionar la resistencia en verde de los cuerpos porosos. Finalmente, éstos se sinterizaron a $1200^{\circ} \mathrm{C}$ empleando una rampa de temperatura de $300^{\circ} \mathrm{C} / \mathrm{min}$. y se sostuvo por 3 horas, con el fin de consolidar la estructura porosa y generar mayor resistencia mecánica [3].

\section{Preparación de las soluciones de quitosano.}

El quitosano comercial fue diluido en ácido acético glacial al $1 \%(\mathrm{v} / \mathrm{v})$ para preparar soluciones poliméricas a diferentes concentraciones $(0,5 \%, 1 \%, 1.5 \%, 2 \% \mathrm{p} / \mathrm{v})$. Todas las soluciones se prepararon con agua destilada a una agitación de $700 \mathrm{rpm}$ en un agitador magnético (BOECO, Germany) por 2 horas a temperatura ambiente.

\section{Obtención de los recubrimientos.}

Los cuerpos porosos utilizados contaron con dimensiones promedio de $3 \mathrm{~mm}$ de radio y $10 \mathrm{~mm}$ de altura, y tuvieron pesos promedio de $0,8 \mathrm{~g}$. dichos cuerpos fueron lavados con etanol al $70 \%(\mathrm{v} / \mathrm{v})$, seguido de un lavado con agua en ultrasonido por $5 \mathrm{~min}$ a $60 \mathrm{~Hz}$ para eliminar el exceso de grasa e impurezas y finalmente se secaron a $70^{\circ} \mathrm{C}$ durante 1 hora, para activar químicamente la superficie.

Los cuerpos activados fueron recubiertos por la técnica de inmersión en $50 \mathrm{ml}$ de solución de Quitosano (Qo) para las diferentes concentraciones evaluadas $(1 \%, 1.5 \%, 2 \% \mathrm{p} / \mathrm{v})$, y se mantuvieron inmersos durante dos tiempos diferentes $(5 \mathrm{y}$ $20 \mathrm{~min}$ ), una vez transcurridos los tiempos de inmersión, fueron secados a $40^{\circ} \mathrm{C}$ en estufa durante 2 horas, con el fin de evaluar la influencia del tiempo de inmersión al generar las diferentes capas de recubrimiento. Luego de la inmersión y el secado, cada muestra se fue lavada con etanol al $70 \%$ y agua destilada para eliminar completamente los residuos y excesos de material.

\section{E. Caracterización de los cuerpos porosos}

La composición química, relación molar $\mathrm{Ca} / \mathrm{P}$ y cristalinidad de los cuerpos con y sin recubrimiento de quitosano se evaluó por Difracción de Rayos X, utilizando un difractómetro de rayos X marca XPert PANalytical con detector Pixel 3D, que usa radiación $\mathrm{CuK} \alpha$ en un rango $2 \theta$ de $5-60^{\circ}$, con velocidad de barrido de $4^{\circ} / \mathrm{min}$ y paso de $0,04^{\circ}$, y la información se complementó con un ensayo de 
Fluorescencia de Rayos X (FRX) utilizando un espectrómetro marca Thermo, modelo Optim'x.

Adicionalmente, se analizaron grupos funcionales y bandas características del Qo y la HA, y la interacción entre los componentes mediante la técnica de Espectroscopía de Infrarrojo con Transformada de Fourier (FTIR). Los espectros de transmisión se consiguieron usando un espectrofotómetro Shimadzu IR AFFINITY-1 en un rango de número de onda $(v)$ entre $400 \mathrm{y} 4000 \mathrm{~cm}^{-1}$.

La caracterización morfológica en cuanto a tamaño de poro, tipo de porosidad e interconectividad de los cuerpos porosos de HA y las características de los recubrimientos de Qo obtenidos con las diferentes concentraciones fue realizada empleando la Microscopía Electrónica de Barrido (SEM), para esto, se empleó un Microscopio electrónico de barrido marca Joel JSM-6490LV con EDS incorporado marca Oxford Instrument (INCA PentaFETx3) para análisis químico, en medio de alto vacío con recubrimiento de oro, y la observación de las muestras se llevó a cabo a diferentes magnificaciones.

La resistencia mecánica de los cuerpos porosos de HA sin recubrimiento y los cuerpos porosos con recubrimientos de Qo en las diferentes concentraciones, se determinó usando ensayos uniaxiales de compresión según la Norma ASTM D882, para lo cual se utilizó una máquina universal de ensayos marca Diggimes con una celda de carga con capacidad para $4900 \mathrm{~N}$ y una velocidad de barrido de $5 \mathrm{~mm} / \mathrm{min}$, con un límite de desplazamiento o deformación hasta el $75 \%$.

\section{RESULTADOS}

\section{A. Caracterización de los cuerpos porosos de HA}

La Fig. 1 muestra la morfología de los cuerpos porosos de hidroxiapatita producidos en el laboratorio de biomateriales por la técnica de gel-casting.

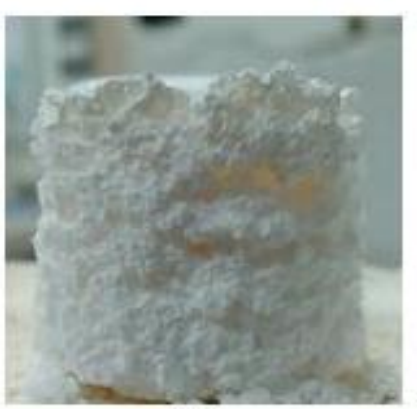

(a)

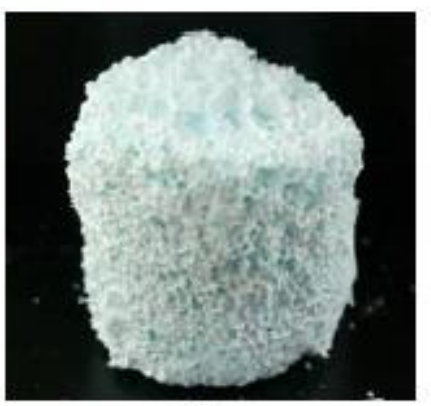

(b)
Fig. 1. Cuerpos de HA con 50\% de sólidos: a) en verde, b) calcinados.

Los cuerpos porosos resultantes presentan una forma adecuada, en la cual se observan poros de tamaño y forma redondeada, homogéneamente distribuidos, y aparentemente interconectados, que le confieren a la estructura una buena resistencia a la manipulación.
El análisis de Fluorescencia de Rayos X (FRX) arrojó la identificación de los compuestos y elementos presentados en la Tabla I, tanto para los cuerpos porosos de hidroxiapatita preparados, como para los patrones de comparación.

TABLA I.

COMPOSICIÓN Química De LOS CuERPos DE HIDROXIAPATITA OBTENIDOS

\begin{tabular}{lcc}
\hline \hline \multicolumn{1}{c}{ COMPUESTO } & CaO & $\mathbf{P}_{\mathbf{2}} \mathbf{O}_{\mathbf{5}}$ \\
\hline Peso (\%) & 62,29 & 46,13 \\
Desviación Estándar & 0,24 & 0,24 \\
ELEMENTO & $\mathbf{C a}$ & $\mathbf{P}$ \\
Peso (\%) & 44,54 & 20,13 \\
Desviación Estándar & 0,17 & 0,17 \\
Moles & 1,11 & 0,65 \\
Relación Ca/P & \multicolumn{2}{c}{} \\
\hline \hline
\end{tabular}

En las Fig. 2 y 3 se presentan los difractogramas de los cuerpos de $\mathrm{HA}$ con y sin recubrimiento de quitosano obtenidos a diferentes concentraciones para 5 y 20 minutos de inmersión.

Las Fig. 2a y 3a muestran los difractogramas de los cuerpos de HA que fueron utilizado como patrón de comparación y los cuerpos de HA recubiertos con quitosano a diferentes concentraciones para 5 y 20 minutos de inmersión respectivamente. El espectro de la muestra patrón muestra una alta cristalinidad de la muestra y evidencia los picos de mayor intensidad en $2 \theta=31.7^{\circ}, 32.2^{\circ}, 32.9^{\circ}$, y otros de menor intensidad en $25.9^{\circ}, 40,46.7^{\circ}$ y $49.4^{\circ}$ que son característicos de la HA, que corresponden para la HA estequiométrica, reportada ampliamente en la literatura por Singh [8] y Boddu [9] entre otros.

Los espectros b, c y d de la Fig. 2 corresponden a los cuerpos de HA recubiertos con Qo a diferentes concentraciones y con 5 minutos de inmersión. Estos espectros presentan zonas más amorfas y con picos de menor intensidad a $10^{\circ}$ y $20^{\circ}$ que son característicos del quitosano con variaciones muy pequeñas en la intensidad, sin embargo, para el cuerpo de HA con $0,5 \%$ Qo (espectro e) no hay presencia del pico a $2 \theta=20^{\circ}$, lo que indica que en la muestra no había suficiente presencia de este polímero, esto puede dar idea que cuando los recubrimientos tiene baja concentración de polímero y tiempos cortos de inmersión, no se logra una adhesión suficiente entre la superficie y el polímero.

Los espectros de la Fig. 3 corresponden a cuerpos de HA recubiertos con Qo a diferentes concentraciones y 20 minutos de inmersión. También en ellos se muestran los picos característicos de quitosano para los cuerpos recubiertos. Para este tiempo, a baja concentración de Qo $(0,5 \%$, espectro c) si se observó un pico en $2 \theta=20^{\circ}$ perteneciente al polímero, lo que indica que a mayor tiempo de inmersión mejora la adhesión del recubrimiento con la superficie. 


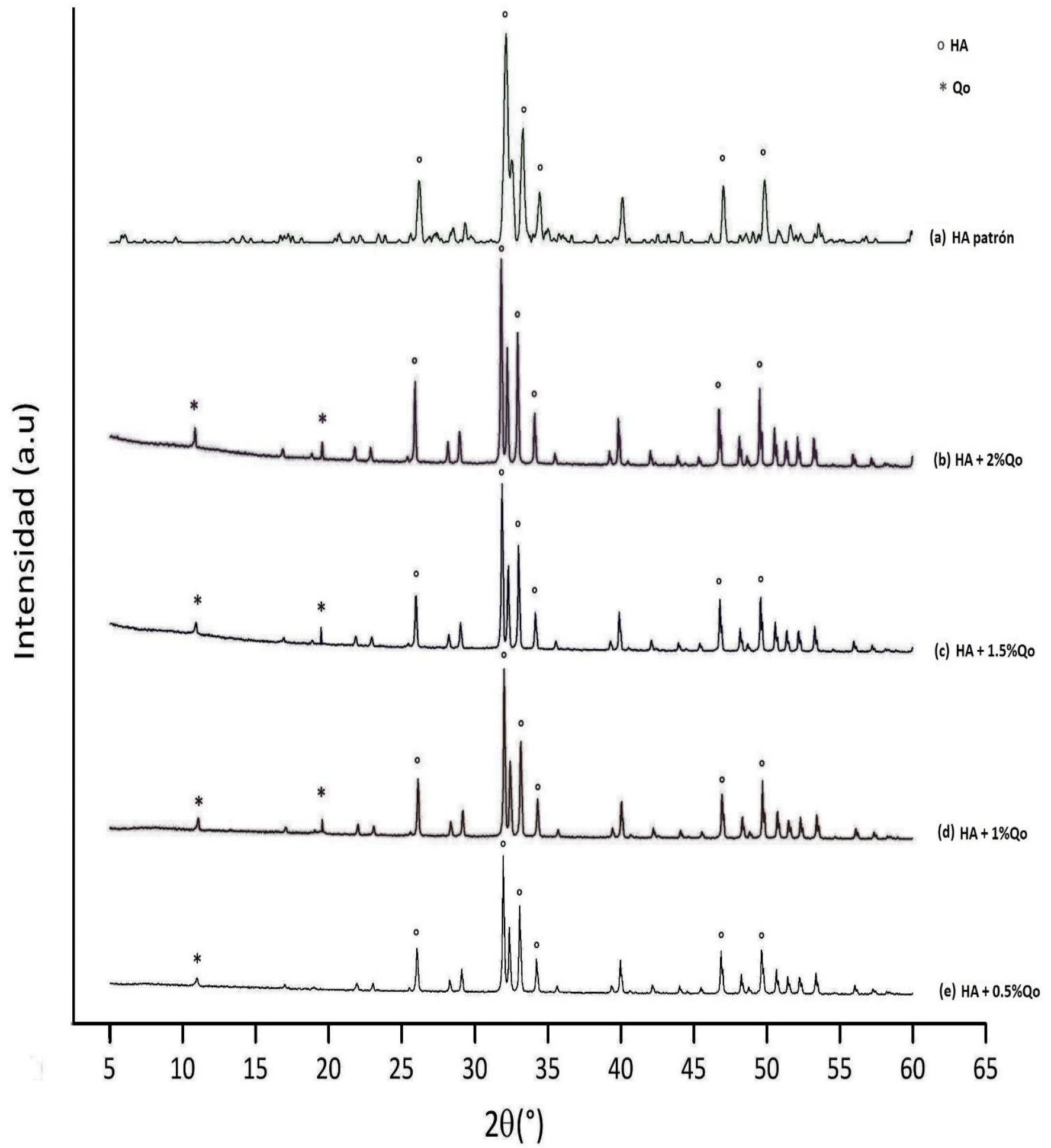

Fig. 2. Difractograma para los cuerpos de HA patrón (a) y los cuerpos recubiertos con $2 \%$ Qo (b), $1,5 \%$ Qo (c), $1 \%$ Qo (d) y $0,5 \%$ Qo (e) para 5 min de inmersión. 


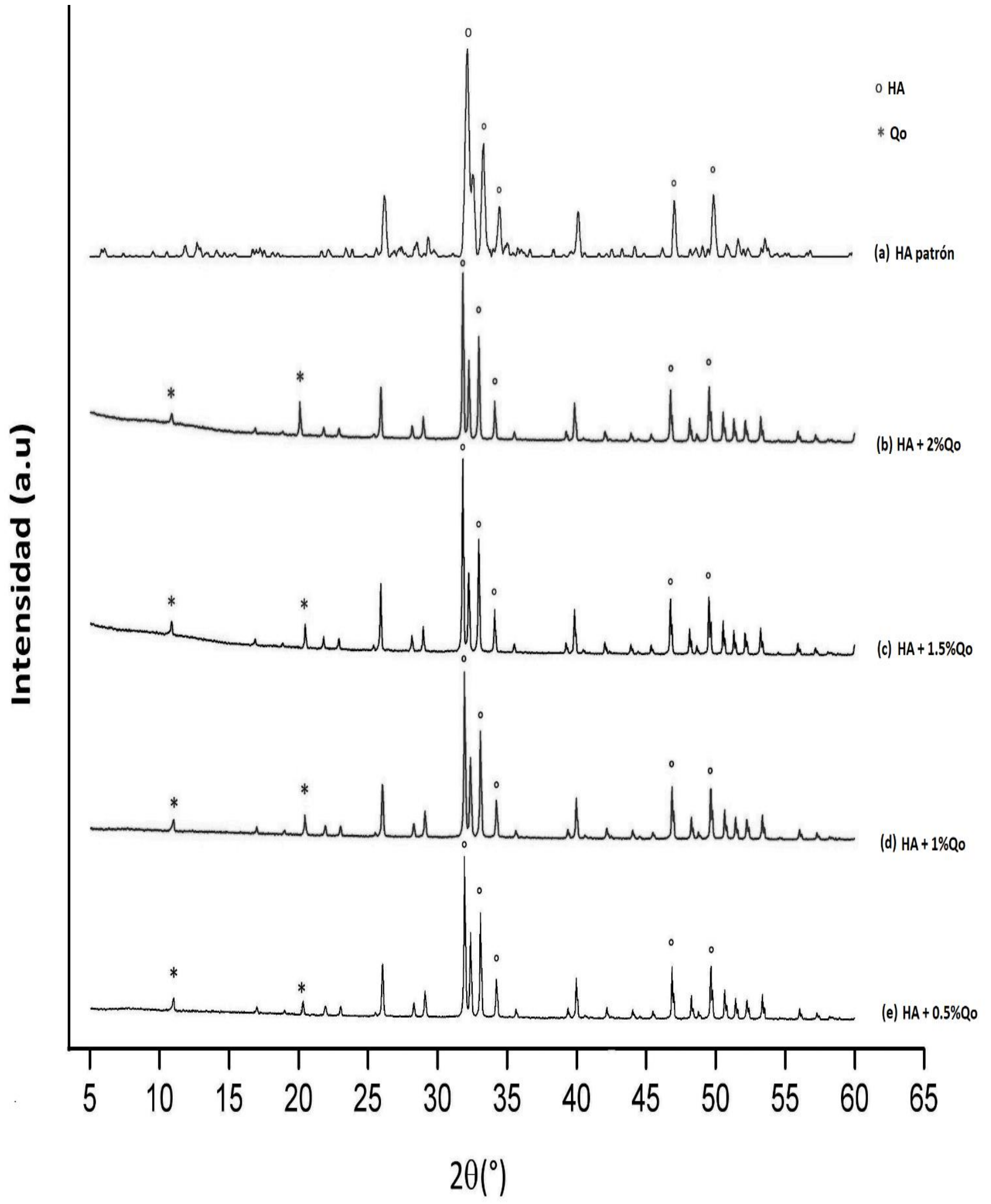

Fig 3. Difractograma para los cuerpos de HA patrón (a) y los cuerpos recubiertos con $2 \%$ Qo (b), 1,5\% Qo (c), $1 \%$ Qo (d) y 0,5\% Qo (e) para 20 min de inmersión 
La cristalinidad de las muestras resulta de la interacción entre cadenas a través de enlaces de hidrogeno y enlaces de van der Waals que se forman entre la superficie de la HA y el Qo (comportamiento catiónico), ya que es una unión de tipo mecánico que se da debido a las cargas de ambos materiales (adhesión electrostática).

Todos los espectros de la Fig. 3, muestran los picos característicos de quitosano para los cuerpos recubiertos, y para $0,5 \%$ Qo (Fig. 3c) a diferencia de la Fig. 3e si se observó un pico en $2 \theta=20^{\circ}$ del quitosano. Esto indica que a mayor tiempo mejora la adhesión del recubrimiento con la superficie y a medida que aumenta la concentración de quitosano la intensidad de los picos también se ve aumentada.
La cristalinidad de las muestras obtenidas resulta de la interacción intermolecular entre cadenas a través de enlaces de hidrogeno intermolecular, enlaces de van der Waals que se forman entre la superficie de la HA y el Qo (comportamiento catiónico), ya que es una unión de tipo mecánico que se da debido a las cargas de ambos materiales (adhesión electrostática).

La Fig. 4 muestra el espectro infrarrojo del quitosano puro utilizado para los recubrimientos (a), los cuerpos de HA patrón (b) y los cuerpos de HA recubiertos con quitosano a diferentes concentraciones y 20 minutos de inmersión.

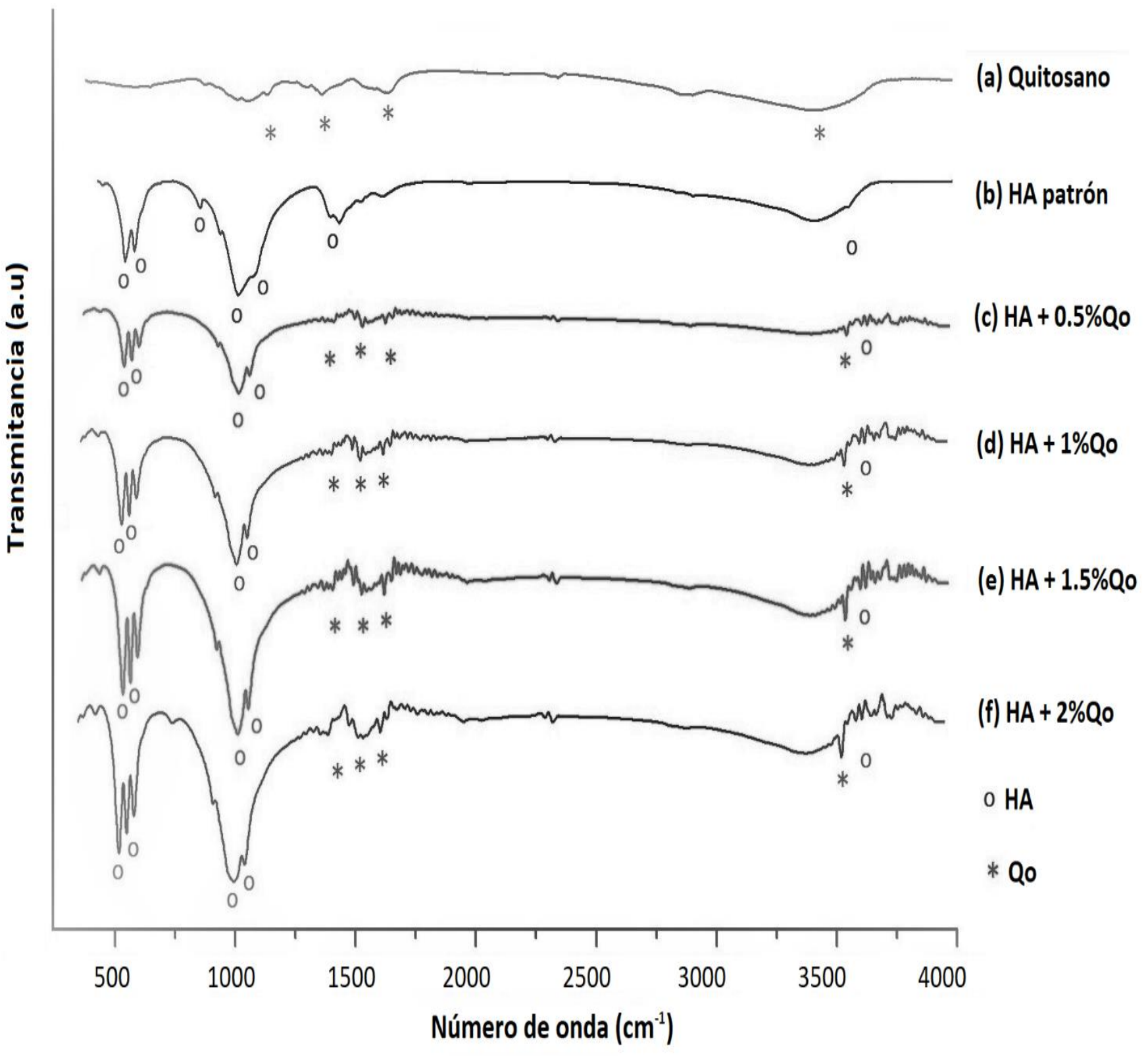

Fig. 1. Espectros obtenidos para el Qo (a), cuerpos de HA (b) y cuerpos de HA recubiertos con Qo a diferentes concentraciones (c - f) para 20 minutos de inmersión 
El espectro de infrarrojo correspondiente al quitosano (espectro a) se logran evidenciar las bandas principales para el quitosano (Qo), bandas correspondientes grupo amida I, observadas en $1630 \mathrm{~cm}^{-1}$, otra banda en $1550 \mathrm{~cm}^{-1}$ atribuida al $\mathrm{NH}_{2}$, en la banda $1200 \mathrm{~cm}^{-1}$ para la amida III y en $3450 \mathrm{~cm}^{-}$ ${ }^{1}$ para el grupo de tensión $\mathrm{OH}^{-}$, estas bandas corresponden a valores muy similares reportados en la literatura por Mesa [10] y Nikpour [11].

En el espectro b se logran observar las bandas características para la HA. Las bandas que vibran alrededor de $630 \mathrm{~cm}^{-1}$ y $3570 \mathrm{~cm}^{-1}$ corresponden al estiramiento de los grupos hidroxilos presentes en la HA o la absorción de agua en la muestra, y las bandas de absorción presentes en 1024 $\mathrm{cm}^{-1}$ y $560 \mathrm{~cm}^{-1}$ corresponden con el estiramiento del grupo $\mathrm{PO}_{4}{ }^{3-}$ en concordancia con lo reportado por Londoño [12] y Budiraharjo [13].

En los espectros de los cuerpos de HA con recubrimiento de Qo a diferentes concentraciones (c-f) se logran observar bandas alrededor de 1580 y $1400 \mathrm{~cm}^{-1}$, características del grupo carboximetil, y las bandas del grupo amina a $1630 \mathrm{~cm}^{-}$ ${ }^{1}$ y la amida a $1550 \mathrm{~cm}^{-1}$, características del Qo. También se observa una reducción en el número de onda del compuesto como resultado de la interacción entre el quitosano y la hidroxiapatita, a través de enlaces de hidrógeno entre $\mathrm{NH}_{2}$ y $\mathrm{OH}^{-}$, así como la quelación entre $\mathrm{NH}_{2}$ y $\mathrm{Ca}^{2+}$ tal y como lo reporta Maji [14].

Puede observarse además que la magnitud de las bandas de transmitancia es más débil y estrecha debido a la hibridación in situ.

La estructura final de las señales en los espectros (c, d, e y f) representa un claro indicio de la elevada estabilidad que posee el recubrimiento obtenido, la cual incrementa con el aumento de la concentración de Qo, como se observa en el espectro. Como era de esperarse todos los picos de HA y Qo son observados para los cuerpos recubiertos acorde con lo reportado por Maji [14] y Tang [15].

En la Fig. 5 se presenta las imágenes de microscopía electrónica de la superficie y el detalle de los poros de uno de los cuerpos de HA utilizado como patrón (sin recubrimiento de quitosano).

En la Fig. 5a se muestra una estructura tridimensional con poros interconectados, homogéneos y de diferentes tamaños, los cuales presentan tamaños superiores a los $200 \mu \mathrm{m}$ debido a la técnica empleada, mientras que en la Fig. 5b se ven algunas partículas aglomeradas, que corresponden a lo reportado en la literatura por Yoshikawa [16], Teixeira [17] y Oliveira [18].

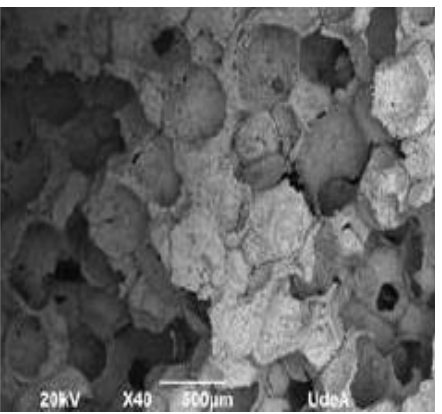

a)

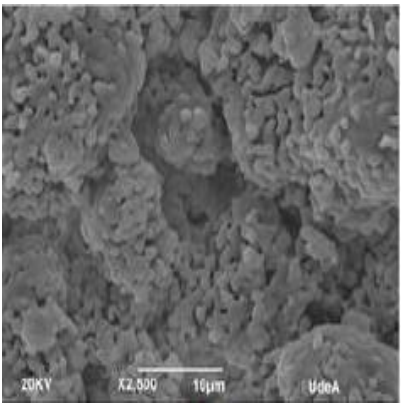

b)
Fig. 5. Micrografía SEM de del cuerpo de HA (a) la superficie y (b) detalle del poro

Las Micrografías de las superficies de los bloques de hidroxiapatita y los detalles de los poros de los cuerpos recubiertos con quitosano al $0.5 \%, 1 \%, 1.5 \%$ y $2 \%$ durante 5 y 20 min de inmersión son presentados en las Fig. 6 y 7 respectivamente.

En ambas Fig. (6 y 7), se observan superficies con estructura porosa e interconectada para todos los cuerpos de hidroxiapatita recubiertos, sin importar la concentración utilizada del recubrimiento. También se puede observar como el recubrimiento polimérico se deposita en forma de película con grumos de material orgánico sobre la superficie de los cuerpos como es el caso del $2 \%$ de Qo.

Además, el biopolímero está distribuido homogéneamente sobre la superficie de los cuerpos sólidos, e incluso logra integrarse dentro de los poros, lo que sugiere una aparente adhesión. Sin embargo, a medida que se incrementa la concentración del biopolímero y el tiempo de inmersión, la capa formada se torna más viscosa y gruesa, y en los cuerpos recubiertos con quitosano al $2 \%$ se alcanza a presentar taponamiento de los poros (Fig. $7 \mathrm{~g}$ ).

Las concentraciones más bajas de quitosano $(0.5 \%$ para ambos tiempos de inmersión) generan películas más delgadas sobre los cuerpos de hidroxiapatita, y dejan algunas zonas desnudas en las que no se evidencia la formación de la película de recubrimiento, lo que indica que hubo poca impregnación o adhesión del biopolímero durante el tiempo de inmersión.

En la Tabla 2 se presentan los resultados de porosidad e interconectividad obtenidos para los cuerpos de hidroxiapatita con y sin recubrimiento de quitosano a diferentes concentraciones y tiempos de inmersión en la solución del biopolímero. 


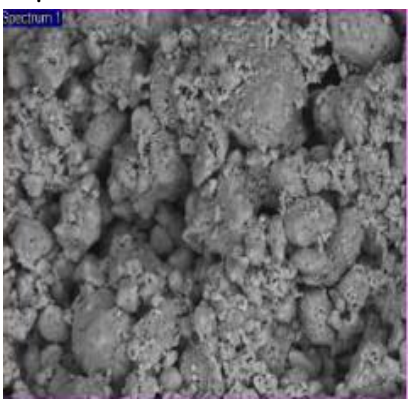

a) Qo $0.5 \%$, superficie

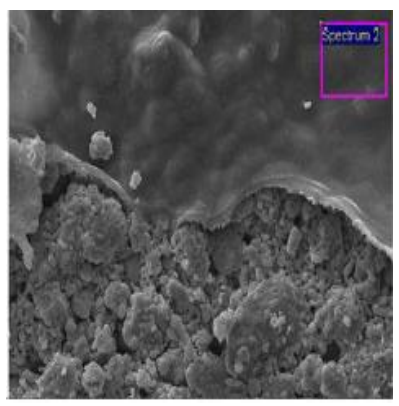

e) Qo $1.5 \%$, superficie

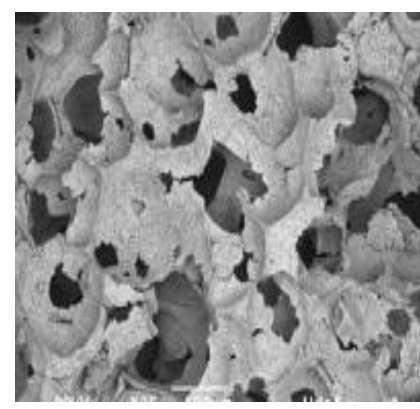

b) Qo $0.5 \%$ poro

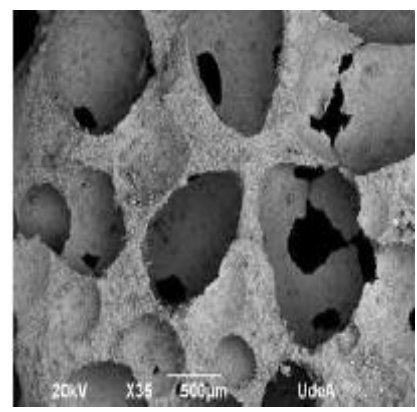

f) Qo $1.5 \%$, poro

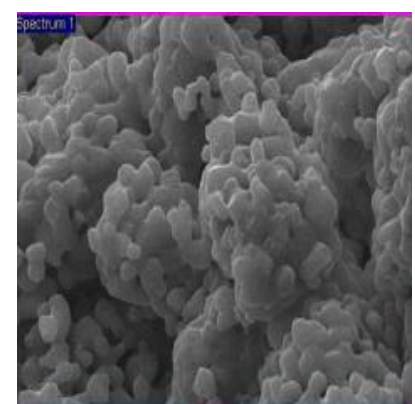

c) Qo $1 \%$, superficie.

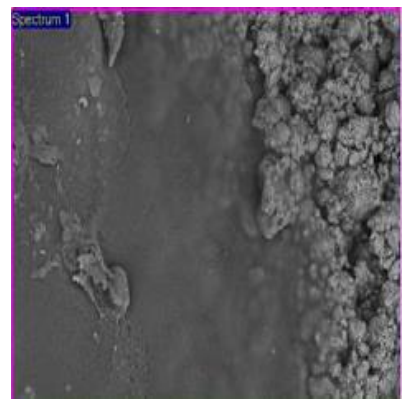

g) Qo 2\%, superficie

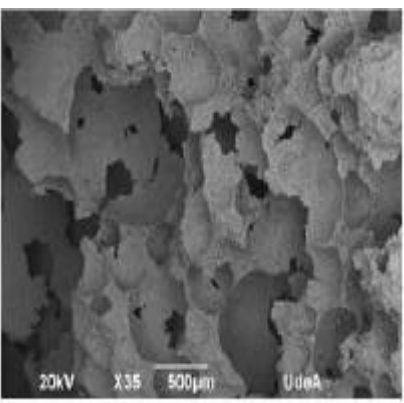

d) Qo $1 \%$, poro

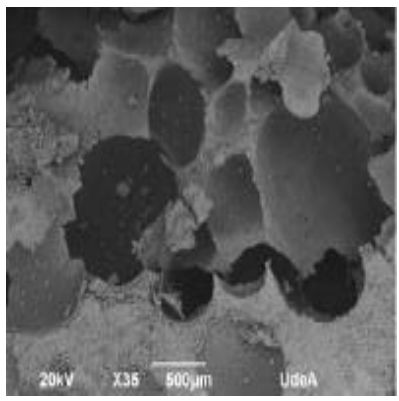

h) Qo $2 \%$, poro

Fig. 6. Imagen de SEM de la superficie y el detalle del poro de los cuerpos de HA recubiertos con quitosano a diferentes concentraciones para 5 min de inmersión

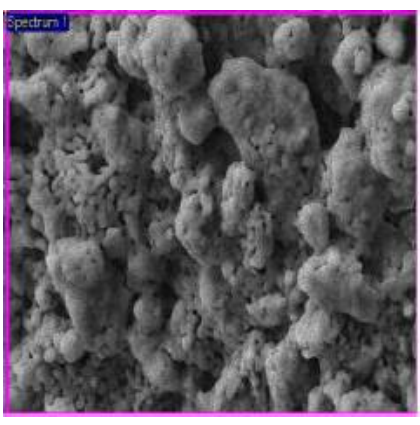

a) Qo $0.5 \%$, superficie

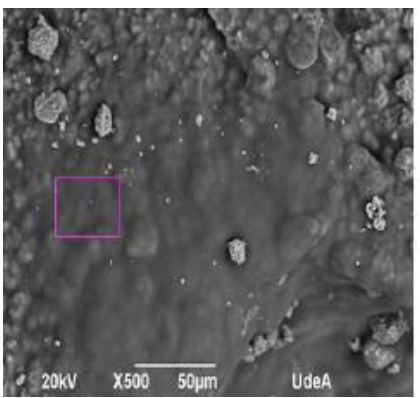

e) Qo $1.5 \%$, superficie

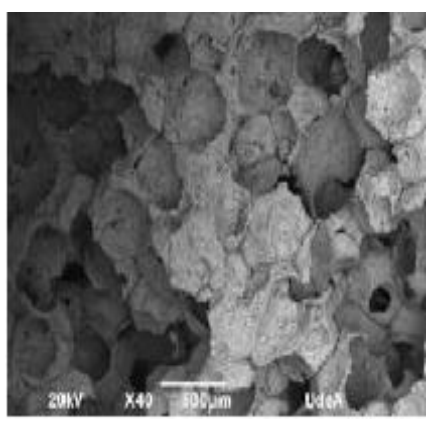

b) Qo $0.5 \%$, poro

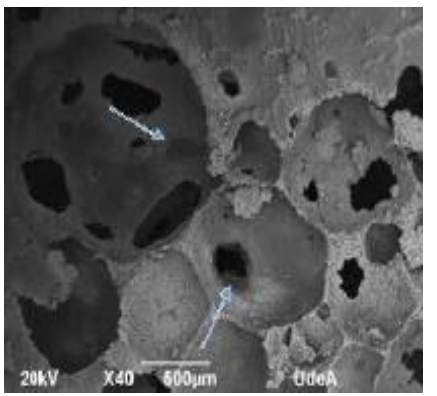

f) Qo $1.5 \%$, poro

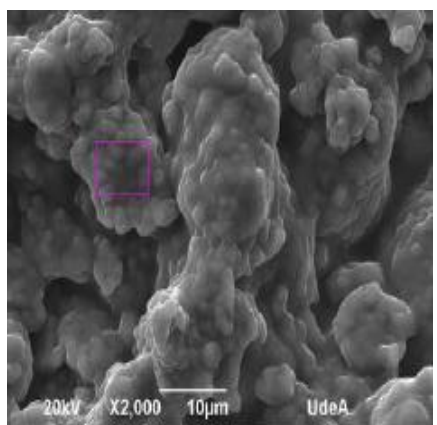

c) Qo $1 \%$, superficie

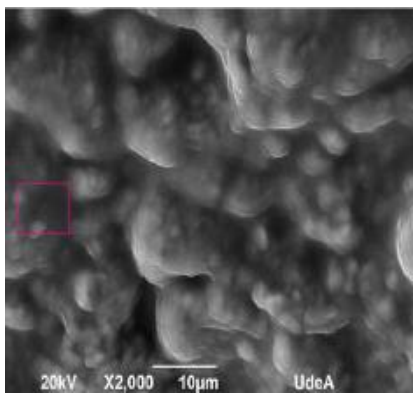

g) Qo $2 \%$, superficie

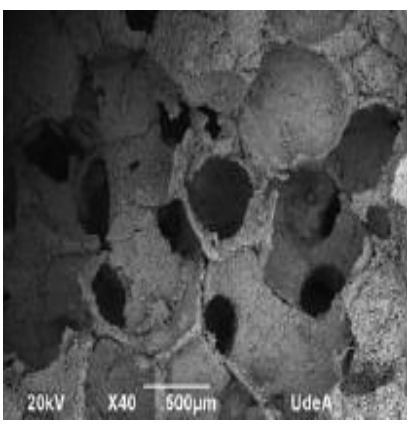

d) Qo $1 \%$, poro

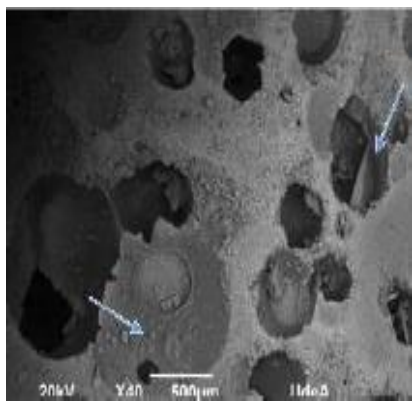

h) Qo $2 \%$, poro

Fig. 7. Imagen de SEM de la superficie y el detalle del poro de los cuerpos de HA recubiertos con quitosano a diferentes concentraciones para 20 min de inmersión 
TABLA II

Porcentaje De Porosidad E InTERConectividad De Los Cuerpos De HA CON Y SIN RECUBRIMIENTO.

\begin{tabular}{|c|c|c|}
\hline Nomenclatura & Porosidad (\%) & $\begin{array}{c}\text { Interconectividad } \\
(\%)\end{array}$ \\
\hline HA patrón & $82,17 \pm 1,0$ & $66,89 \pm 7,8$ \\
\hline $0,5 \%$ Qo $-5 \min$ & $80,34 \pm 1,2$ & $68,15 \pm 7,8$ \\
\hline $0,5 \%$ Qo $-20 \mathrm{~min}$ & $76,40 \pm 0,2$ & $69,55 \pm 0,8$ \\
\hline $1 \%$ Qo $-5 \mathrm{~min}$ & $79,24 \pm 0,1$ & $54,86 \pm 0,2$ \\
\hline $1 \%$ Qo $-20 \mathrm{~min}$ & $75,46 \pm 5,3$ & $53,73 \pm 1,6$ \\
\hline $1,5 \%$ Qo $-5 \mathrm{~min}$ & $83,42 \pm 0,6$ & $54,72 \pm 4,8$ \\
\hline $1,5 \%$ Qo $-20 \mathrm{~min}$ & $78,78 \pm 3,1$ & $46,52 \pm 4,4$ \\
\hline $2 \%$ Qo $-5 \mathrm{~min}$ & $78,15 \pm 7,0$ & $54,99 \pm 7,6$ \\
\hline $2 \% \mathrm{Qo}-20 \mathrm{~min}$ & $83,67 \pm 0,1$ & $49,39 \pm 1,2$ \\
\hline
\end{tabular}

En la Tabla II se compara la porosidad lograda para los cuerpos porosos con y sin recubrimiento. Todos presentaron porosidad entre el 75,46 y $83,67 \%$ e interconectividades entre el 46,52 y $69,55 \%$, y tamaño de poro semejante antes y después de recubrirlos.

Aunque el tamaño de poro obtenido es pequeño, los valores alcanzados se encuentran dentro de los rangos reportados por autores como Boccaccini [19], Hutmacher [20], Kim [21] y González [22, 23], quienes obtuvieron porcentajes de porosidad entre 40 y $92 \%$ para cuerpos porosos preparados con 50 y $60 \%$ de sólidos, demostrando que el recubrimiento realizado tiene influencia en este parámetro, ya que se observa una disminución en la interconectividad a medida que aumenta la concentración de quitosano y el tiempo de inmersión.

\section{B. Evaluación mecánica de los cuerpos porosos}

Las Fig. 8 y 9 muestran las curvas de esfuerzo-deformación obtenidas para los cuerpos de HA sin y con recubrimiento de Qo para diferentes tiempos de inmersión.

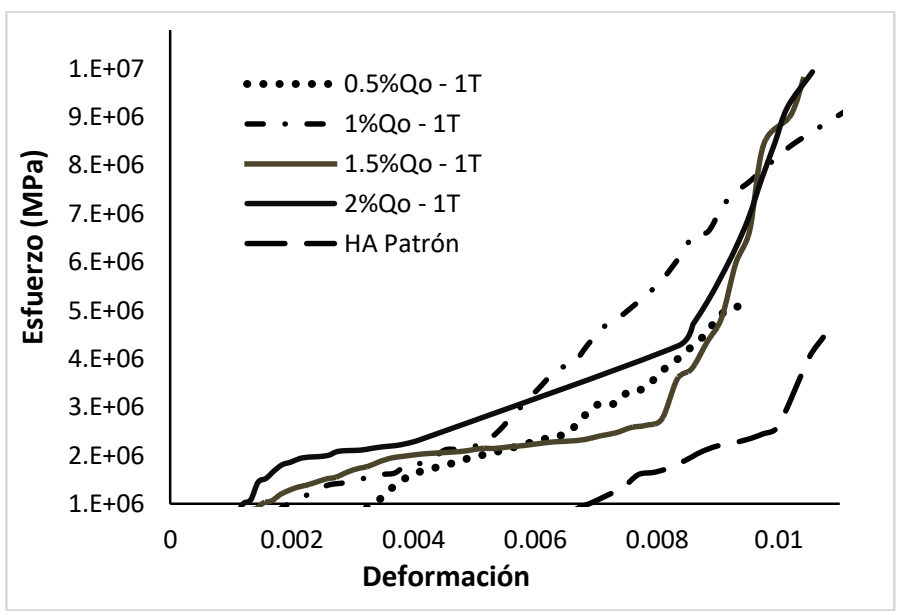

Fig. 8. Curvas de esfuerzo-deformación para cuerpos de HA con y sin recubrimiento para 5 min de inmersión

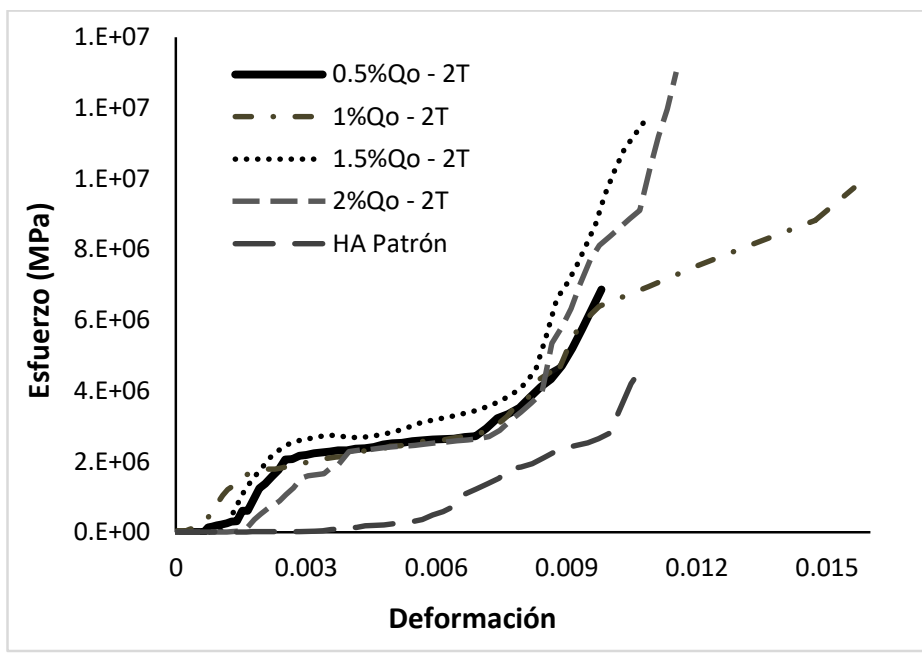

Fig. 9. Curvas de esfuerzo-deformación para cuerpos de HA con y sin recubrimiento para 20 min de inmersión.

A su vez, en la Tabla III se resumen los datos promedio obtenidos de las curvas de Esfuerzo- deformación para la resistencia a la compresión y el módulo de elasticidad para cada uno de los scaffolds evaluados y su respectiva desviación estándar.

TABLA III.

PROPIEDADES MECÁNICAS DE LOS SCAFFOLDS DE HA CON Y SIN RECUBRIMIENTOS DE QO

\begin{tabular}{cccc}
\hline \hline $\begin{array}{c}\text { Quitosano } \\
(\mathbf{\%})\end{array}$ & $\begin{array}{c}\text { Tiempo } \\
\text { inmersión } \\
(\mathbf{m i n})\end{array}$ & $\begin{array}{c}\text { Resistencia } \\
\text { Máxima }(\boldsymbol{\sigma})\end{array}$ & $\begin{array}{c}\text { Módulo Elástico } \\
(\mathbf{M P a})\end{array}$ \\
\hline $\mathbf{0 , 5}$ & 5 & $3,72 \pm 1,55$ & $601,40 \pm 26,07$ \\
$\mathbf{0 , 5}$ & 20 & $5,27 \pm 1,66$ & $470,15 \pm 33,64$ \\
$\mathbf{1 , 0}$ & 5 & $5,76 \pm 3,40$ & $845,00 \pm 53,35$ \\
$\mathbf{1 , 0}$ & 20 & $6,34 \pm 3,20$ & $623,17 \pm 24,51$ \\
$\mathbf{1 , 5}$ & 5 & $7,54 \pm 2,70$ & $1005,32 \pm 46,91$ \\
$\mathbf{1 , 5}$ & 20 & $7,77 \pm 2,99$ & $897,39 \pm 22,64$ \\
$\mathbf{2 , 0}$ & 5 & $8,07 \pm 2,12$ & $1452,27 \pm 38,74$ \\
$\mathbf{2 , 0}$ & 20 & $9,80 \pm 2,73$ & $2929,71 \pm 48,71$ \\
patrón & --- & $2,68 \pm 1,04$ & $287,99 \pm 36,30$ \\
\hline \hline
\end{tabular}

Los recubrimientos de quitosano con menor concentración de Qo y tiempo de inmersión tienen una resistencia a la compresión más baja, pero mayor capacidad de deformación, esta propiedad se ve mejorada en los recubrimientos con mayor concentración de biopolímero y a mayores tiempos de inmersión, debido a que demuestran aumento tanto en la rigidez como en la resistencia mecánica y una leve disminución en el módulo elástico.

Según algunos reportes hechos por Navarro [24] y Diggs [25], la resistencia a la compresión para el hueso trabecular varía entre 2,2 y $10 \mathrm{MPa}$ y el módulo elástico entre 400 y $1700 \mathrm{MPa}$. La resistencia mecánica para la mayoría de los cuerpos de HA recubiertos con Qo alcanzaron valores dentro de dichos rangos, incluyendo los cuerpos patrón, sin 
embargo, sus valores son muy bajos con respecto a los otros, por lo que cabe resaltar que recubrimientos a porcentajes inferiores al $2 \%$ se pueden usar para mejorar la resistencia, según lo observado en el valor de Módulo elástico, para la muestra patrón y la muestra con $2 \%$ Qo para 20 min de inmersión.

Como se observa en la Tabla 3 la mayoría de los scaffolds de hidroxiapatita con recubrimiento de quitosano tienen valores de resistencia mecánica dentro del rango reportado en la literatura incluyendo los scaffolds patrón (sin recubrimiento), sin embargo, sus valores son muy bajos con respecto a los otros, por lo que cabe resaltar que la capa de polímero funcionó de manera positiva con respecto a esta propiedad observándose un aumento en estos valores para los scaffolds con concentraciones de Qo y tiempos de inmersión mayores.

Las desviaciones estándar en general no son muy altas para estos valores lo cual significa que los datos están menos dispersos y que se encuentran más cercanos al promedio. Sin embargo, al observar el Módulo de elasticidad (Y), se muestra que para los scaffolds de HA patrón, los valores fueron muy bajos con respecto a los scaffolds con recubrimientos de Qo y para los scaffolds con mayor concentración de $2 \%$ Qo y tiempo de inmersión $20 \mathrm{~min}$, ambos valores difieren en un buen porcentaje con respecto a los valores reportados para hueso trabecular, esta propiedad los hace poco atractivos para su uso en ingeniería de tejidos.

La determinación de estas propiedades mecánicas es útil a la hora de comparar las condiciones de obtención del material y permite seleccionar mejor un recubrimiento para una aplicación concreta. En este estudio se han evaluado la resistencia máxima y el módulo de elasticidad de scaffolds de HA con recubrimientos de Qo a diferentes concentraciones obtenidos por inmersión a dos tiempos (5 y $20 \mathrm{~min}$ ).

Se han observado importantes diferencias respecto al valor que se obtiene para los sustratos de HA sin recubrimientos. El daño en los ensayos se produce de forma importante a través de los poros que lo constituyen, indicando que éstas son zonas de unión relativamente débiles, debido a la presencia de un recubrimiento polimérico que ayuda a mejorar la cohesión y actúa como una especie de adhesivo estas propiedades o resistencia al daño pudieron ser mejoradas.

Dada la propia naturaleza de este tipo de recubrimientos, sus espesores y a la anisotropía de las muestras, hace que sus propiedades mecánicas sean difíciles de determinar, sin embargo, se han relacionado las propiedades mecánicas analizadas con la estructura de los recubrimientos.

\section{CONCLUSIONES}

La adición de recubrimiento de quitosano sobre los cuerpos de HA logró mejorar la resistencia a la compresión, y aunque la alteración de la microestructura (tamaño e interconectividad) se vio afectada por el taponamiento de los poros con el aumento la concentración de quitosano, todavía se evidencia potencialidad en aplicación para Ingeniería de Tejido óseo.

Los recubrimientos de quitosano se desarrollan como una alternativa prometedora para preservación y conservación de estructuras tridimensionales con ventajas de bajo costo y facilidad de procesamiento.

La obtención de recubrimientos de quitosano sobre cuerpos de hidroxiapatita con el método de inmersión ha sido poco estudiado, lo que resulta interesante debido a que se logra obtener información relevante de propiedades como la adhesividad y el tipo de interacción que presenta el polímero sobre sustratos cerámicos de hidroxiapatita, de la cual no se encuentra mucha información en la literatura.

\section{AGRADECIMIENTOS}

Las autoras desean expresar sus a agradecimientos a la Universidad de Antioquia y en especial al Grupo de Investigación en Biomateriales de la Facultad de Ingeniería, por financiar el proyecto del cual se derivó esta publicación.

\section{REFERENCIAS}

[1] C. Martínez, A. Ozols. "Biomateriales utilizados en cirugía ortopédica como sustitutos del tejido óseo". Revista de la Asociación argentina de ortopedia y traumatología. 77 (2), pp: 140-146, octubre, 2012. DOI:10.15417/43

[2] G. Wei, P. Ma. "Macroporous and nanofibrous polymer scaffolds and polymer/bone-like apatite composite scaffolds generated by sugar spheres". Journal of Biomedical Materials Research -Part A, 78(2): pp. 306-315, Aug, 2006. DOI:10.1002/jbm.a.30704

[3] J. González, D.M. Escobar, C. Ossa. "Manufacturing and characterization of hydroxyapatite foams produced by gel-casting technique". Health Care Exchanges. pp.1-6. May, 2013. DOI:10.1109/PAHCE.2013.6568289

[4] C. Carter, M. Norton. "Ceramic Materials: Science and Engineering. Springer Science \& Business Media", 2 ed. Springer, New York. 766 p. 2013. ISBN 978-1-4614-3523-5

[5] C. Peniche, Y. Solís, N. Davidenko, R. García. "Materiales compuestos de quitosano e hidroxiapatita". Biotecnología Aplicada, 27(3), pp: 192-201, julio, 2010.

[6] S. Mishra, S. Kannan. "Development, mechanical evaluation and surface characteristics of chitosan/polyvinyl alcohol based polymer composite coatings on titanium metal". Journal of the mechanical behavior of biomedical materials. 40, pp: 314-324, December, 2014. DOI: 10.1016/j.jmbbm.2014.08.014

[7] D. Goloshchapov V. Kashkarov N. Rumyantseva P. Seredin, A. Lenshin B. Agapov, E. Domashevskaya. "Synthesis of 
nanocrystalline hydroxyapatite by precipitation using hen's eggshell”. Ceramics International, 39 (4) pp.4539-4549, May 2013. DOI: $10.1016 /$ j.ceramint.2012.11.050

[8] A. Singh. "Hydroxyapatite, a biomaterial: Its chemical synthesis, characterization and study of biocompatibility prepared from shell of garden snail", Helix aspers. Bulletin of Materials Science. 35 (6) pp: 1031-1038, November, 2012. https://www.ias.ac.in/article/fulltext/boms/035/06/1031-1038

[9] V. Boddu, E. Smith. "Composite biosorbent for treatment of waste aqueous system(s) containing heavy metals". Patente US6786336 B2. United States, September, 2004.

[10] N. Mesa, S. Ospina, D.M. Escobar, D. Rojas, P. Zapata, C. Ossa. "Isolation of chitosan from Ganoderma lucidum mushroom for biomedical applications". Journal of Materials Science: Materials in Medicine 26 (135), pp:1-9, March, 2015. DOI:10.1007/s10856-15-5461-z

[11] M. Nikpour, S. Rabiee, M. Jahanshahi. "Synthesis and characterization of hydroxyapatite/chitosan nanocomposite materials for medical engineering applications". Composites Part B: Engineering. 43(4), pp: 1881-1886., June, 2012. DOI: 10.1016/j.compositesb.2012.01.056

[12] M. Londoño, A. Echavarría, F. De La Calle. "Características cristaloquímicas de la hidroxiapatita sintética tratada a diferentes temperaturas". Revista EIA, 5 pp:109-118, junio, 2006. http://www.scielo.org.co/pdf/eia/n5/n5a10.pdf

[13] R. Budiraharjo, K. Neoh. E. Kang. "Hydroxyapatite-coated carboxymethylchitosan scaffolds for promoting osteoblast and stem cell differentiation". Journal of Colloid and Interface Science 366 (1), pp: 224-232. January, 2012. DOI: 10.1016/j.jcis.2011.09.072

[14] Maji K. and Dasgupta S., "Hydroxyapatite-Chitosan and Gelatin Based Scaffold for Bone Tissue Engineering". Transactions of the Indian Ceramic Society. 73 (2), pp: 110-114, april, 2014. DOI: 10.1080/0371750X.2014.922424

[15] S. Tang, B. Tian, Y. Guo, Z. Zhu. "Chitosan/carbonated hydroxyapatite composite coatings: Fabrication, structure and biocompatibility". Surface \& Coatings Technology 251, Pp:.210216, July, 2014. DOI: 10.1016/j.surfcoat.2014.04.028

[16] Yoshikawa, N. Tamai, T. Murase, A. Myoui. "Interconnected porous hydroxyapatite ceramics for bone tissue engineering". Journal of the Royal Society Interface. 6 (3) pp.341-348, December 2008. DOI:10.1098/rsif.2008.0425.focus

[17] S. Teixeira, M. Rodriguez, P. Pena, A. De Aza, M. Ferraz, F. "Monteiro. Physical characterization of hydroxyapatite porous scaffolds for tissue engineering". Materials Science and Engineering: C, 29 (5) pp: 1510-1514, June 2009. DOI: 10.1016/j.msec.2008.09.052

[18] J. Oliveira, S. Silva, P. Malafaya, M. Rodrigues, M. Gomes, R. Reis. "Macroporous hydroxyapatite scaffolds for bone tissue engineering applications: Physicochemical characterization and assessment of rat bone marrow stromal cell viability". Journal of Biomedical Materials Research Part A.- 91 (1), pp: 175-186, October 2009. DOI:10.1002/jbm.a.32213

[19] A. Boccaccini, X. Chatzistavrou, Y. Mohamad, V. Califano. "Biodegradable polymer-bioceramic composite scaffolds for bone tissue engineering. Department of Materials", Imperial College London, London SW7 2BP, UK. June, 2009.

[20] D. Hutmacher. "Scaffolds in tissue engineering bone and cartilage”. Biomaterials. 21(24). pp.2529-2543. December, 2000. DOI: 10.1016/S0142-9612(00)00121-6

[21] H. Kim, S. Lee, C. Bae, Y. Noh, H. Kim, J. Ko. "Porous $\mathrm{ZrO}_{2}$ bone scaffold coated with hydroxyapatite with fluor apatite intermediate layer". Biomaterials 24 (19), pp. 3277-3284, August, 2003. DOI: 10.1016/S0142-9612(03)00162-5
[22] J. González, D.M. Escobar, C. Ossa. "Influence of the Type of Manufacturing Technique on the Porosity and Interconnectivity of Hydroxyapatite Scaffolds". International Journal of Materials Engineering Innovation. 7(2), pp: 104-114, September, 2016. DOI: 10.1504/IJMATEI.2016.079554

[23] J. González, D.M. Escobar, C. Ossa. "Porous bodies of hydroxyapatite produced by a combination of the gel-casting and polymer sponge methods". Journal of Advanced Research. 7 (2), pp: 297-304, March, 2016. DOI: https://doi.org/10.1016/j.jare.2015.06.006

[24] M. Navarro. "Desarrollo y Caracterización de Materiales Biodegradables para Regeneración Ósea”. Universitat Politècnica de Catalunya. Departamento de Ciencia de los Materiales, Julio, 2005.

https://upcommons.upc.edu/bitstream/handle/2117/93360/01Mnt 01de11.pdf

[25] A.B Diggs. Influence of Calcium Phosphate Composition and Desing on Bone Regeneration, Degradation and Mechanical Function. Ph.D. dissertation. Biomedical Engineering. University of Michigan. 2013. http://hdl.handle.net/2027.42/97853

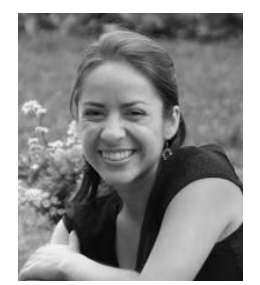

Natali Mesa Ospina, es graduada Bioingeniera en el año 2012 y de Magister en Ingeniería de Materiales en 2017 de la Universidad de Antioquia.

Ella hizo parte del Grupo de Investigación en biomateriales de la Universidad de Antioquia desde 2011 cuando era estudiante del Programa de Bioingeniería, participando en proyectos de investigación relacionados con la producción de hidroxiapatita y posteriormente trabajó con la extracción de biopolímeros desde fuentes naturales como el quitosano desde los caparazones de crustáceos.

Actualmente se desempeña como Representante de Ventas para el área de neuro intervencionismo y cardiopatías congénitas de la Empresa Interventional Medical Products, en el Departamento de Santander-Colombia.

ORCID: http://orcid.org/0000-0001-5317-6366

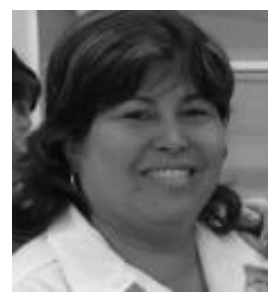

Diana Marcela Escobar Sierra recibió su título de Ingeniera Metalúrgica en 1996 y se tituló como Ph.D en Ciencias Químicas en el año 2003.

Ella participó en Grupos de investigación en materiales atmosféricos como el Grupo de corrosión y posteriormente en el año 2003cuando se vinculó al Programa de Bioingeniería, creó el Grupo de Investigación en Biomateriales. 
Actualmente es Profesora Titular de la Facultad de Ingeniería en la Universidad de Antioquia y es la Coordinadora del Grupo de Investigación en Biomateriales -BIOMAT- donde se desempeña en las áreas de síntesis y caracterización de cerámicos, y la extracción de biopolímeros desde fuentes naturales, ambos para aplicaciones médicas y cosmetológicas.

ORCID:http://orcid.org/0000-0002-6013-7039 